\title{
Las cenas navideñas de empresa. ¿Un modelo de consumo recreativo de alcohol y otras drogas?
}

\section{Christmas work dinners. A pattern of recreational use of alcohol and other drugs?}

\author{
ANTONIO VIDAL-INFER*, ,**, SOFÍA TOMÁS-DOLS ${ }^{\star \star *}$, \\ Remedios Aguilar-Moya*, Teresa Samper-Gras*, \\ María J. Zarza *, Julia Aguilar-Serrano*
}

* Fundación para el Estudio, Prevención y Asistencia a las Drogodependencias. Conselleria de Sanitat. Generalitat Valenciana;

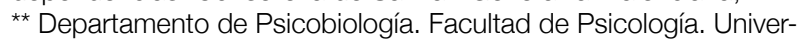
sitat de València;

*** Dirección General de Drogodependencias. Conselleria de Sanitat. Generalitat Valenciana

Enviar correspondencia a:

Antonio Vidal Infer. Facultad de Psicología. Dpto. Psicobiología Universitat de València Avda. Blasco Ibáñez 2146010 Valencia.

Correo-e.: Antonio.Vidal-Infer@uv.es

\section{RESUMEN}

El objetivo del presente estudio fue realizar una aproximación exploratoria para caracterizar el patrón de consumo recreativo abusivo de alcohol y otras drogas que se da en las cenas navideñas de empresa. Para ello se ha empleado una adaptación de la metodología de estimación rápida RARE, mediante la combinación de técnicas cuantitativas (encuestas de estimación rápida, $n=444$ ) y cualitativas (observaciones y entrevistas semiestructuradas, $n=8$ ). El trabajo de campo se desarrolló en 13 restaurantes de las ciudades de Valencia y Alicante (España) durante el mes de diciembre de 2007. En líneas generales se percibe un notable aumento del consumo de alcohol y otras sustancias en comparación al resto del año, si bien este año el consumo abusivo de alcohol, según los hosteleros, ha bajado respecto a años anteriores debido a la legislación vigente en materia de controles de tráfico. La cocaina es la sustancia ilegal percibida como más consumida en estos eventos. Los jóvenes inician en el consumo de sustancias ilegales a los adultos, quienes utilizan dicha conducta como vía de rejuvenecimiento y desinhibición. Las cenas navideñas de empresa propician un patrón de consumo recreativo que requiere de medidas preventivas y restrictivas adaptadas, tales como la puesta en marcha de medios de transporte adecuados para estos eventos y la restricción de horarios para el servicio de alcohol.

Palabras clave: etnografía, cenas de Navidad, estimación rápida, alcohol, abuso de drogas

\section{ABSTRACT}

The aim of this study was to develop an exploratory approach to characterizing the pattern of recreational abuse of alcohol and other drugs abuse at Christmas work dinners. An adaptation of the rapid assessment methodology RARE was carried out through the combination of quantitative (rapid assessment surveys, $n=444$ ) and qualitative (observations and semistructured interviews, $n=8$ ) techniques. The fieldwork was carried out at 13 restaurants in Valencia and Alicante (Spain) in December 2007. In general we observed a marked increase in the use of alcohol and other substances compared to the rest of the year, though restaurant staff perceived a decrease in alcohol abuse attributable to new road traffic legislation. Cocaine is perceived as the illicit substance most widely used at such events. Younger coworkers initiate older ones in the use of illicit drugs, and the the latter may use this behaviour as a means of feeling younger and more uninhibited. Christmas work dinners propitiate a recreational substance-use pattern that requires adapted preventive and restrictive interventions, such as the introduction of suitable transport initiatives and the restriction of alcohol serving hours.

Key words: ethnography, Christmas work dinners, rapid assessment, alcohol, drug abuse 


\section{INTRODUCCIÓN}

E concepto de consumo recreativo de drogas ha surgido en los últimos años para caracterizar el uso de sustancias psicoactivas en adolescentes y jóvenes adultos que se produce fundamentalmente durante los fines de semana'. Una de las actividades más características de la juventud española es salir los fines de semana, y es entonces cuando algunos de ellos toman contacto con el alcohol y las drogas ilegales, haciendo de su consumo una pauta imprescindible de las salidas nocturnas. Numerosos estudios relacionan el consumo de alcohol y otras drogas con los fines de semana y la población juvenil ${ }^{2,3,4,5}$, en lo que se ha definido como un consumo recreativo de drogas ${ }^{6}$. En líneas generales, los jóvenes que consumen alcohol, salvo casos muy concretos, lo hacen exclusivamente durante los viernes, sábados y/o domingos ${ }^{7}$. A este consumo de alcohol hay que unir, en muchos casos, el consumo de otras drogas ilegales ${ }^{8}$. Se trata, por tanto, de consumos que se dan en grandes cantidades y concentrados en un breve periodo de tiempo, con el objetivo de buscar desinhibición y nuevas sensaciones ${ }^{9}$. Sin embargo, este tipo de consumo no sólo se da en un segmento de población joven durante los fines de semana. En los últimos años se ha instaurado en España una tradición en la que muchas empresas organizan una fiesta de navidad para sus empleados, consistente en una comida o cena en un restaurante, en que se suelen producir consumos abusivos tanto de comida como de alcohol y otras sustancias adictivas. Estas celebraciones se han convertido en los últimos años en un evento muy concurrido; de hecho, la previsión en la Comunidad Valenciana fue de aproximadamente 200.000 cenas en la campaña navideña del año $2007^{10}$.

La literatura científica respecto al consumo de sustancias psicoactivas en celebraciones navideñas es escasa. Se han realizado estudios de campo para comparar los niveles de intoxicación etílica en determinadas celebraciones anuales, tales como Halloween o el día de San Patricion", eventos conocidos en Estados Unidos por el gran consumo de alcohol asociado a su celebración. Otros trabajos, a su vez, han puesto de manifiesto las consecuencias asociadas a los incrementos del consumo abusivo de alcohol en las diferentes celebraciones puntuales, especialmente las navideñas, tales como las arritmias del "Holiday Heart" o sindrome del corazón post-vacacional ${ }^{12,13,14,15,16}$, la pancreatitis ${ }^{17}$, y las muertes por intoxicación etílica ${ }^{18,19,20,21,22}$. Apenas existen estudios que relacionen el incremento de consumo abusivo de otro tipo de drogas durante estos eventos con la problemática que conlleva, aunque sí que se ha encontrado relación entre el consumo abusivo puntual de cannabis y el sindrome del corazón post-vacacional ${ }^{23}$.

Aunque su nombre parezca indicar que se trata de un consumo no problemático, el consumo recreativo de drogas puede acarrear importantes problemas para los individuos a largo plazo, y estas implicaciones pueden suponer un importante problema de salud pública que requiera intervenciones específicas adaptadas a la población. Todavía no se ha estudiado con detalle el patrón de consumo de alcohol $y$, especialmente, de otras sustancias adictivas, en este tipo de celebraciones, pese a que cualquier labor preventiva en materia de drogodependencias debe partir del conocimiento, percepción y actitudes de los agentes implicados. Es necesario, por tanto, realizar una aproximación exploratoria para caracterizar el consumo recreativo de sustancias psicoactivas durante estos eventos.

\section{MÉTODO}

El estudio se realizó aplicando el modelo RARE de Estimación Rápida, Intervención y Evaluación ${ }^{24,25}$, que consiste en la utilización de un conjunto integrado de técnicas cuantitativas y cualitativas que, mediante la triangulación de la información obtenida, permite la identificación de patrones de comportamiento ${ }^{26}$. La presente investigación ha contado con tres técnicas para contrastar tres perspectivas diferentes del fenómeno de las cenas de Navidad, como son la del comensal, la del experto en hostelería y la del propio trabajador de campo.

El trabajo de campo se desarrolló entre los días 12 y 22 de diciembre de 2007. En este período de tiempo se llevaron a cabo observaciones durante las noches, entre las $21 \mathrm{~h}$ y la hora de cierre, en diversos restaurantes de las ciudades de Valencia y Alicante. Tras las observaciones, se administraron encuestas de estimación rápida en el exterior de dichos restaurantes. Por último, se realizaron entrevistas semiestructuradas de tipo confirmatorio a informadores clave procedentes del mundo de la hostelería. Para llevar a cabo el trabajo de campo, se formaron tres equipos de dos personas. Uno de los equipos realizó las observaciones y las encuestas de estimación rápida en la ciudad de Alicante, y los otros dos desarrollaron su trabajo en Valencia. Los trabajadores de campo recibieron previamente formación especifica en observación exploratoria y administración de encuestas.

A continuación, se describen cada una de las técnicas utilizadas para recopilar la información.

\section{Técnicas}

\section{Observación exploratoria. La perspectiva del investigador.}

El registro de las observaciones permitió caracterizar los rituales de consumo de alcohol y otras drogas antes, durante y después de la cena, así como identificar los diferentes roles que asumen los miembros de cada mesa, y que puedan tener algún tipo de relación con el consumo de sustancias adictivas. Los restaurantes $(N=13)$ se seleccionaron según un procedimiento de muestreo intencional por variación máxima ${ }^{27}$ para conseguir la mayor información posible con la más alta representatividad. Se escogieron según diferentes dimensiones de interés, tales como la localización geográfica, el precio medio del cubierto y el tipo de comida ofertada (Tabla 1). 
Tabla 1. Descripción de los restaurantes donde se realizó el trabajo de campo.

\begin{tabular}{cccc}
\hline Código & Ciudad & Precio medio cubierto & Tipo comida \\
\hline R01 & Valencia & $>30 €$ persona & Asador \\
R02 & Valencia & $>30 €$ persona & Asador \\
R03 & Alicante & $15-30 €$ persona & Tapas/Bocadillos \\
R04 & Valencia & $15-30 €$ persona & Tapas/Bocadillos \\
R05 & Valencia & $>30 €$ persona & Tapas/Bocadillos \\
R06 & Valencia & $15-30 €$ persona & Tapas/Bocadillos \\
R07 & Valencia & $15-30 €$ persona & Pizzeria \\
R08 & Alicante & $15-30 €$ persona & Pizzeria \\
R09 & Valencia & $15-30 €$ persona & Tapas/Bocadillos \\
R10 & Valencia & $>30 €$ persona & Asador \\
R11 & Alicante & $15-30 €$ persona & Tapas/Bocadillos \\
R12 & Valencia & $>30 €$ persona & Asador \\
R13 & Valencia & $15-30 €$ persona & Pizzeria \\
\hline
\end{tabular}

Encuestas de estimación rápida. La opinión de los comensales.

Tras completar las observaciones, los trabajadores de campo realizaron encuestas de estimación rápida mediante el método "Street Intercept"28, 29, 30, a individuos presentes en las zonas próximas a los restaurantes. La muestra estuvo compuesta por 444 sujetos, de los que el $54,6 \%$ venian de una cena de empresa frente a un $45,4 \%$ que no, con un $57,3 \%$ de hombres y un $42,7 \%$ de mujeres, y una edad media de 33,36 años ( $d t=10,332)$. Respecto a su situación laboral, entre otras ocupaciones, el 56,2\% eran empleados, el 15,8\% estudiantes, y el 15,1\% autónomos.

El cuestionario utilizado estaba compuesto por seis ítems, con modalidades de respuesta tanto abierta como cerrada. Las dimensiones evaluadas fueron: a) percepción de consumo de sustancias, incluyendo la más frecuente, b) comparación respecto al consumo en otras ocasiones o noches "normales", c) caracterización del consumidor y del tipo de consumo, d) relación posterior con zonas de ocio, incluyendo desplazamientos y conducción, y e) consumo propio de sustancias y su comparación respecto al consumo en otras ocasiones o noches "normales". Cabe añadir que durante el diseño del cuestionario se decidió diferenciar el alcohol de los otros tipos de sustancias.

\section{Entrevistas semiestructuradas.}

El discurso de los profesionales de la hostelería.

Tras el trabajo de campo, se realizaron entrevistas semiestructuradas a informadores clave, entendiendo como tales aquellas personas que proporcionan información experta y en profundidad sobre los elementos de una cultura, sociedad o escenario social ${ }^{31}$. En este caso, se entrevistó a ocho profesionales del ámbito de la hostelería, cuyos perfiles se describen en la tabla 2.
Tabla 2. Perfil de los entrevistados.

\begin{tabular}{|c|c|c|c|}
\hline Código & Descripción del cargo & Sexo y edad & Localización \\
\hline E01 & Jefe de Sala & Hombre, 34 años & Alicante \\
\hline E02 & Propietario y camarero & Hombre, 37 años & Alicante \\
\hline E03 & $\begin{array}{c}\text { Representante de } \\
\text { agrupación de } \\
\text { hosteleros }\end{array}$ & Hombre, 45 años & C. Valenciana* \\
\hline E04 & $\begin{array}{l}\text { Propietario y jefe } \\
\text { de sala }\end{array}$ & Hombre, 50 años & Valencia \\
\hline E05 & Jefa de sala & Mujer, 31 años & Valencia \\
\hline E06 & $\begin{array}{l}\text { Propietaria y jefa } \\
\text { de sala }\end{array}$ & Mujer, 45 años & Valencia \\
\hline E07 & Propietario y camarero & Hombre, 45 años & Valencia \\
\hline E08 & $\begin{array}{l}\text { Propietario y jefe } \\
\text { de sala }\end{array}$ & Hombre, 60 años & Valencia \\
\hline
\end{tabular}

(*) Entrevista realizada en la ciudad de Valencia, si bien el ámbito de trabajo de la agrupación a la que representa incluye toda la Comunidad Valenciana (Castellón, Valencia y Alicante)

La duración aproximada de cada entrevista osciló entre 25 y 60 minutos. Las dimensiones incluidas en el guión de la entrevista fueron las siguientes: a) cumplimiento de las expectativas hosteleras en cuanto al consumo de alcohol, b) caracterización del consumo de alcohol en las cenas navideñas de empresa, c) perfil del consumidor abusivo, d) el consumo de sustancias ilegales, e) perfil del consumidor de sustancias ilegales, f) diferencias respecto a una noche normal de fin de semana, g) rutas posteriores de ocio, y h) el papel del profesional de la hostelería en futuras intervenciones preventivas.

\section{Análisis de datos}

\section{Análisis estadistico}

El análisis de la información procedente de las encuestas de estimación rápida fue llevado a cabo mediante el software estadístico SPSS v.15. Se realizó un análisis descriptivo y de frecuencias, y posteriormente se desarrolló un análisis bivariado de asociación entre las variables relevantes del estudio. La significación estadistica fue establecida en una $\mathrm{p}<0.05$.

\section{Análisis cualitativo}

El procedimiento de análisis cualitativo de las observaciones y las entrevistas se basó en los actuales modelos de estimación rápida ${ }^{32}$, mediante la Teoría Fundamentada o Grounded Theory ${ }^{33}$. La estrategia establecida en el estudio para el desarrollo de los conceptos y temas clave fue la del Método Comparativo Constante ${ }^{34}$, que fue utilizada para codificar y analizar los datos simultáneamente, con tal de desarrollar los hallazgos y las recomendaciones eventuales. 


\section{Integración de la información}

Por último, mediante un proceso de triangulación, se combinaron los resultados de todas las técnicas utilizadas en la investigación, de manera que cada dimensión emergente en los datos era contrastada según las tres perspectivas existentes, para dotar a la información de una mayor profundidad

En todos los casos se informó a los participantes sobre los objetivos del estudio y el anonimato y confidencialidad de los datos obtenidos.

\section{RESULTADOS}

Tras el análisis de los datos y la posterior triangulación de la información resultante, se han destacado nueve dimensiones de análisis diferenciadas, cuyas características principales se recogen en la tabla 3 .

\section{Caracterización de las cenas navideñas de empresa}

Estas celebraciones se han consolidado como un evento de confraternización organizacional, especialmente entre los distintos niveles jerárquicos de la empresa (...es precisamente el jefe el primero que necesita hacer de la cena de empresa ese día de aproximación a sus trabajadores... (E03)).
Según las observaciones y el discurso de los profesionales hosteleros, el tipo de ambiente que se puede encontrar en una cena depende del contexto donde se realice (...depende del tipo de restaurante que hagas la observación verás un tipo de gente y un tipo de consumo u opiniones... (0:R03)). Además, los grupos que asisten a cenas navideñas de empresa se caracterizan por su heterogeneidad (...una mesa que parece ser cena de empresa por la diferencia de edades y estilos de vestir y conversación... (0:R03)). El número de comensales varía en función del tamaño de la empresa, aunque suelen ser eventos muy concurridos (...la media son veinte, de veinte a treinta personas... (E05)).

El consumo de alcohol y otras sustancias aumenta en estas fechas, al tratarse de una ocasión especial (...como es una fiesta que hacen una vez al año y se hace en esas fechas, pues la gente se alarga más en el consumo... (E04)), y por el propio ambiente de celebraciones festivas (...se bebe más en Navidad que en cualquier cena, eh... (EO6)). Además, al tratarse de eventos sufragados económicamente por la propia empresa, en muchas ocasiones se aprovecha para realizar consumos mayores de lo habitual (...cuando son comidas y cenas de empresa, si que el consumo de alcohol es mayor, por el esto de que no lo pago yo, pues..., como es gratis... (EO4)), aunque para algunos, el consumo abusivo de las cenas de Navidad es un fenómeno puntual, que tenderá a la baja en los próximos años (...después de haber pasado por un boom, yo creo que eso es normal que, si lo sabemos manejar, se vaya moderando... (EO3)).

Tabla 3. Dimensiones de análisis y resultados más destacados.

\begin{tabular}{|c|c|}
\hline DIMENSIÓN & CARACTERÍSTICAS \\
\hline Caracterización de las cenas de Navidad & 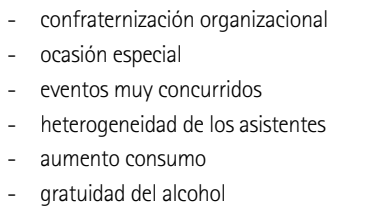 \\
\hline Consumo de alcohol & $\begin{array}{l}\text { - secuencia definida } \\
\text { a. espera (vino y cerveza) } \\
\text { b. entrantes (vino y cerveza) } \\
\text { c. desarrollo (vino y cerveza) } \\
\text { d. después (cava, licores y combinados) }\end{array}$ \\
\hline Consumo de sustancias ilegales & $\begin{array}{l}\text { - cenas de Navidad, puerta de entrada al } \\
\text { consumo de sustancias ilegales } \\
\text { - cocaina, alta percepción de prevalencia } \\
\text { de consumo } \\
\text { - difícil detección de consumo de sustan- } \\
\text { cias ilegales }\end{array}$ \\
\hline Iniciación en el consumo & $\begin{array}{l}\text { - consumen incluso los no consumidores } \\
\text { - identificación factores de riesgo: } \\
\text { a. actitudes y normas favorables hacia } \\
\text { la conducta } \\
\text { b. disponibilidad de la droga } \\
\text { c. baja resistencia a la frustración } \\
\text { d. estatus socioeconómico } \\
\text { e. rechazo por parte de iguales }\end{array}$ \\
\hline Instrumentalización del consumo & $\begin{array}{l}\text { - via de rejuvenecimiento } \\
\text { - } \quad \text { búsqueda de sensaciones }\end{array}$ \\
\hline
\end{tabular}

\begin{tabular}{|c|c|}
\hline DIMENSIÓN & CARACTERÍSTICAS \\
\hline Perfil del consumidor & $\begin{array}{l}\text { - } \text { diferente al del consumidor recreativo de } \\
\text { - } \text { fin de semana } \\
\text { - } \text { - estigmatización } \\
\text { - } \text { equiparación respecto al tipo de alcohol } \\
\text { consumido } \\
\text { - } \quad \text { existencia de un rol de catalizador del } \\
\text { consumo }\end{array}$ \\
\hline Subagrupaciones & $\begin{array}{l}\text { - } \quad \text { formación de subgrupos por afinidad } \\
\text { o evitación } \\
\text { - } \quad \text { mayor desinhibición } \\
\text { - } \quad \text { mayor consumo de alcohol } \\
\text { - } \quad \text { no relación con consumo de sustancias } \\
\text { ilegales }\end{array}$ \\
\hline Alcohol y tráfico & $\begin{array}{l}\text { - } \quad \text { reducción del consumo de alcohol } \\
\text { - poca concienciación respecto a otras } \\
\text { sustancias }\end{array}$ \\
\hline Propuesta de medidas preventivas & $\begin{array}{l}\text { - medios de transporte adecuados para } \\
\text { _ } \quad \text { mayos espentos } \\
\text { seguridad del Estado sobre detección de } \\
\text { sustancias ilegales } \\
\text { - } \quad \text { restricción de horarios para el servicio } \\
\text { de alcohol } \\
\text { _ } \\
\text { subida de precios del alcohol }\end{array}$ \\
\hline
\end{tabular}




\section{El consumo de alcohol}

La secuencia del consumo de alcohol comienza con la espera del resto de los comensales (... a la espera de más gente, se consume cervezas, refrescos y agua... (0:R02)) y los entrantes, donde las bebidas alcohólicas predominantes son el vino y la cerveza (...comienzan pidiendo la bebida, la mayoría consumen vino salvo alguna excepción que bebe agua o cerveza... (0:R12)). Éstas son, además, las bebidas que predominan en el desarrollo de la cena (...están cenando cuando entramos y en la mesa se visualizan botellas de agua y jarras de cerveza. También hay alguna botella de vino... (0:R04)). Tras los cafés, es el momento de las bebidas alcohólicas de mayor graduación (... ya han terminado de cenary piden poleos para después algunos beben cubatas de whisky... (0:R02)). Este patrón se repite en el discurso de todos los entrevistados (..., el aperitivo y una cerveza o dos y un vermú o lo que sea. Luego ya viene el vino... Y luego ya la copa. Bueno, y por supuesto el cava o el champán, que también tiene su lugarcito... (E04)).

En referencia al alcohol, los encuestados durante el trabajo de campo percibian un mayor consumo coincidente con estas celebraciones, ya que el 99,1\% de los mismos manifestó que en las cenas navideñas de empresa se producian consumos de alcohol, y un 75,1\% percibía que se consumía más o mucho más que en el caso de una noche normal de fin de semana. Tras la cena, el consumo de alcohol continúa en otros lugares de ocio (...luego, cuando terminan aqui en el restaurante, se van de fiesta también. 0 sea, que el consumo sigue... (E04)).

\section{El consumo de sustancias ilegales}

Los profesionales de la hostelería identificaron las cenas de Navidad como puerta de entrada al consumo de drogas ilícitas (...el problema no es la cena de empresa, sino la cena de empresa como puerta de entrada a problemas... (E03)). Según la percepción de los encuestados, un 46,9\% manifestó que en estos eventos se llevaban a cabo conductas de consumo de sustancias ilegales, y de éstos, un 66,9\% consideraba que se consumía más o mucho más que en una noche cualquiera de un fin de semana. Las sustancias que se suelen consumir, según éstos, son el alcohol $(80,4 \%)$, cocaína $(52,7 \%)$, cannabis $(38,3 \%)$, crystal $(9,7 \%)$, éxtasis $(9 \%)$, alucinógenos $(6,1 \%)$ y otros tipos de sustancias menos comunes $(1,1 \%)$, mientras que la droga ilegal que se percibe como más consumida es la cocaína $(71,4 \%)$, seguida del cannabis $(25,1 \%)$.

La percepción de la cocaína como sustancia ilegal más consumida es compartida por los profesionales entrevistados (...hay coca en las cenas de empresa y que ese es un comentario que circula... (EO3)), si bien se trata de una sustancia cuya percepción social negativa, fundamentalmente debida al mayor peligro percibido con respecto a otras sustancias como el cannabis ${ }^{35}$, hace que su consumo no goce de la aprobación del resto de los miembros (...pasa un poco desapercibido, sobre todo cuando son cenas de empresa, porque es un hábito que está prohibido y mal visto también... (E01)) y, por tanto, es más difícil de detectar (...la gente intenta evi- tar ese consumo, hasta que salga fuera del restaurante o de la cena y ya sean otro tipo de grupo, o del mismo grupo... (E01)), e incluso es difícil que, pese a haber consumido, los encuestados lo reflejen en los cuestionarios (...hay dos personas de cena de empresa que no contestan a cuestionario pero nos dicen que han consumido alcohol y drogas durante la cena, están de juerga... (0:R11)).

Sin embargo, ni en el trabajo de campo ni tras las entrevistas con los hosteleros se identificó la presencia de cannabis en estos eventos (...hachis y marihuana son muy difíciles de detectar... (E01)), ya que se trata, probablemente, de la sustancia en la que es más difícil ocultar tanto su consumo (...casi todo el local es no fumador, entonces no se puede fumar hachis o marihuana porque está prohibido fumar ya de por sí... además huele muchísimo... (E01)) como sus síntomas (...si te pones delante de una persona y hablas con ella, se lo detectas... (E01)).

Según los entrevistados, la identificación por parte de los trabajadores del ámbito hostelero del consumo de sustancias es posible (...el que está en los códigos de ese tipo de conductas, evidentemente las identifica inmediatamente, el que no, pues no... (E03)), aunque el ritmo de trabajo no siempre permite disponer del tiempo ni la atención necesarios para fijarse (...tengo muchas cosas que hacer como para estar pendiente de quien se levanta, de quien bebe más cerveza de lo normal... (E02)). Uno de los representantes hosteleros define tres tipos de perfiles de percepción desde la perspectiva del responsable del establecimiento (...la percepción que se puede tener desde los establecimientos de hostelería pasa por estos tres tipos de perfiles, el que lo identifica, el que no lo identifica, y el que se hace el loco... (E03)).

Respecto a la percepción de los encuestados sobre los lugares donde se producen los consumos, la mayoría identificaba el propio espacio del restaurante $(27,8 \%)$ y sus aseos $(24,4 \%)$ como los puntos donde se consumian las sustancias, mientras que un 21,1\% manifestó que los consumos se llevaban a cabo en pubs y zonas de ocio próximas. El trabajo de campo corroboró la percepción de los encuestados, ya que existían indicios de consumo de cocaina en los baños y aseos (...a esta hora un varón de aproximadamente 30 años se va al baño y se pasa allí un buen rato, al salir se limpia la nariz y aspira... (0:R06)).

Sin embargo, los profesionales manifiestan discursos contradictorios, que van desde la identificación de sospechosas visitas en grupo al cuarto de baño (...en alguna ocasión si que he visto que ciertas personas, siempre las mismas, van siempre al baño... (E02);...uno detrás de otro. Uno primero, otro después... y bueno el orden... No van en grupo, van de uno en uno... (EO4)) a la no identificación de claves o indicios de consumo de sustancias ilegales (...me imagino que alguna vez, alguien pues habrá entrado a esnifar lo que sea, pero es indetectable... (E07)).

El análisis de las encuestas demostró que no existian diferencias estadisticamente significativas en cuanto a la relación entre el consumo de drogas y el haber asistido a una cena navideña de empresa (Tabla 4). 
Tabla 4. Consumo de drogas y presencia en cenas navideñas de empresa*.

\begin{tabular}{|c|c|c|c|c|}
\hline & & \multicolumn{3}{|c|}{ ¿Vienes de una cena de Navidad? } \\
\hline & & $\mathrm{Si}$ & No & Total \\
\hline \multirow{3}{*}{$\begin{array}{l}\text { ¿Has consumido drogas } \\
\text { esta noche? }\end{array}$} & Si & $8(3,5 \%)$ & $11(5,8 \%)$ & $19(4,6 \%)$ \\
\hline & No & $218(96,5 \%)$ & $178(94,2 \%)$ & $396(95,4 \%)$ \\
\hline & Total & $226(100 \%)$ & $189(100 \%)$ & $415(100 \%)$ \\
\hline
\end{tabular}

*No se registraron diferencias estadisticamente significativas

\section{La iniciación en el consumo}

En este tipo de eventos, experimentan con sustancias ilegales incluso aquellas personas que no consumen sustancias (...gente que no consume, termina consumiendo esa noche porque se ha tomando tres copas y ese tipo de cosas... (E03)), algo que también ocurre con el alcohol (...síque veo gente que bebe solamente, exclusivamente esas fechas, y no está acostumbrada a beber durante el año... (E06)). En el caso de estas cenas, se han detectado tras el trabajo de campo diversos factores que pueden provocar el inicio en los consumos de sustancias:

a. Leyes y normas favorables hacia la conducta (....verdaderamente a la hostelería no le interesa, porque los mayores beneficios que saca es en alcohol... (E05)).

b. Disponibilidad de la droga (...las cenas de empresa han servido de autovía de acceso a la cocaína para la población que no estaba en contacto con sustancias ilegales... (E03)).

c. Baja resistencia a la frustración (...creo que se puede llegar a producir una curiosidad sobre... "yo no quiero ser carroza, no quiero quedarme fuera del grupo"... (E03)).

d. Estatus socioeconómico (...es población con un alto poder adquisitivo y que de alguna manera, cuando quiera consumir cocaína no tendrá ningún problema... (E03)).

e. Rechazo por parte de los iguales (...gente mayor, con un escaso nivel de integración en el grupo, gente muy rutinaria... (E03)).

Además, los encuestados señalaban la búsqueda de sensaciones $(24,9 \%)$, la desinhibición $(1,6 \%)$, la presión grupal $(25,4 \%)$ y que se trata de una ocasión señalada $(12,4 \%)$ como razones para experimentar con nuevas sustancias.

\section{La instrumentalización del consumo}

Tal y como se ha relatado en el apartado anterior, según los entrevistados, en este tipo de eventos hay casos en que personas que habitualmente no consumen aprovechan la ocasión para hacerlo, utilizando la sustancia y los efectos de su consumo como un instrumento para conseguir un objetivo: a. El consumo como vía de rejuvenecimiento. En el caso de estas cenas, son los más jóvenes quienes propician el inicio en el consumo de sustancias de aquellos con una edad superior quienes, a su vez, asumen el consumo como una manera fácil y rápida de sentirse joven y aceptado en el grupo (...es el día del año en que igual se hace unas rayas con gente joven... (E03)).

b. La progresiva desinhibición de los asistentes (...cuando es cena de empresa, que parece que todo vale, que se grita más, que no les importa quién haya delante... (E02)).

\section{Perfil del consumidor}

Los datos de la Encuesta Domiciliaria sobre consumo de drogas en la Comunidad Valenciana del año $2006^{36}$ ponen de manifiesto que el consumo abusivo de alcohol, cannabis, cocaína y drogas de sintesis es más frecuente en un rango de edad de entre 15 y 34 años, y suele darse durante los fines de semana en zonas de ocio. En las cenas navideñas de empresa, el perfil de consumidor no siempre se identifica como el consumidor recreativo joven de fin de semana. En este caso, y de manera puntual, las características son diferentes (...vida familiar, separado, gente mayor... que de repente ese día, son el centro de atención de la reunión... (E03)).

Los encuestados corroboran una aparente predominancia masculina en el consumo de sustancias psicoactivas, ya que un $82,7 \%$ de los hombres había consumido alcohol esa noche, frente a un $74,2 \%$ de las mujeres, mientras que en el caso del consumo de otras drogas, el porcentaje de hombres que habian consumido era más de tres veces mayor $(6,4 \%)$ que el de las mujeres $(1,8 \%)$, siendo las diferencias estadisticamente significativas $(p<0,05)$ en ambos casos (Figura 1).

Los hosteleros también identifican esta mayor prevalencia de consumo en hombres (...normalmente, los chicos

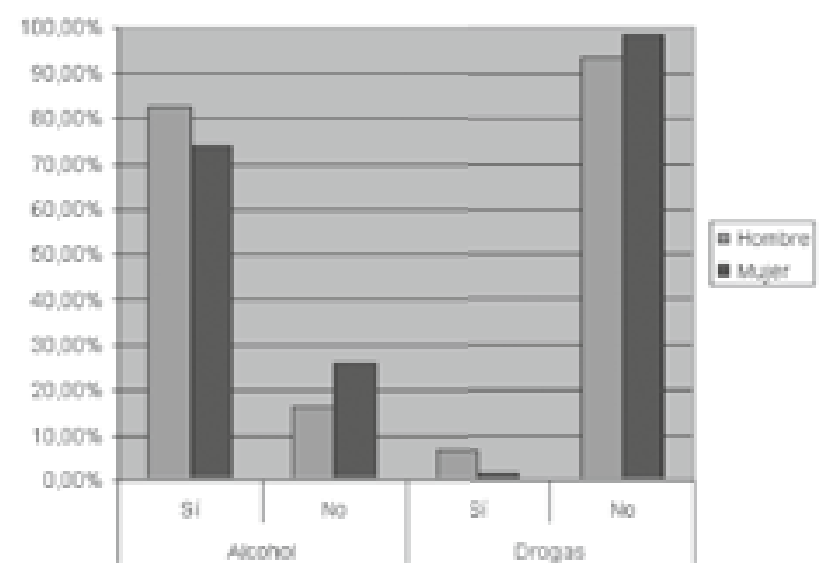

Figura 1. Consumo de alcohol y otras drogas según sexo. 
suelen consumir normalmente más que las chicas... (E07)), y alguno de ellos atribuye estas diferencias al consumo oculto en mujeres (...el tema del consumo femenino es un hecho evidente, pero la mujer sigue teniendo una cierta discreción... (E03)).

Pese a las diferencias observadas respecto a la cantidad de consumo, los trabajadores de la hostelería no destacaron ninguna diferencia entre sexos respecto al tipo de alcohol consumido (...hoy día, no hay diferencias entre sexo, puede ser, porque las mujeres y los hombres más o menos, toman el mismo tipo de alcohol... (EO4)), ni respecto al consumo de sustancias ilegales (... ahí no hay separación de sexos, no... (E04)). Sin embargo, sí que indican diferencias en el consumo con relación a la edad del consumidor (...la gente cuanto más joven es, pues más bebe... (E07)).

Tanto en el trabajo de campo como según el discurso de los entrevistados, se puede distinguir el perfil de ciertos comensales que ejercen un rol de catalizadores del consumo, fundamentalmente de alcohol (...ese mismo que llena la copa, al terminar la jarra, se la llena él y a los amigos y luego te dice, "tráeme otra, corre", es el mismo que enseguida te pide la otra... (E02)). También ejerce dicho rol tras la cena, a la hora de las bebidas de mayor graduación (...lo más curioso es que si uno se la pide, se la piden todos... (E05)). Los hosteleros definen el perfil del catalizador como una persona extravertida y despierta (...pues el de la empresa que es el más espabilado, como siempre. No sé como decirlo, el líder... (E05);... el espabilado o el listillo de todos... que va llenando las copas siempre... (E06)), con una edad en torno a los cuarenta años (...yo creo que es una persona mayor... mediana edad... no es un joven de veintipico, que es de cuarenta,... (E06)), y suelen ser varones (...suelen ser más chicos que chicas, sí, los que desempeñan ese papel... (E07)), si bien alguno de los entrevistados indica que no existe un perfil concreto de catalizador (...puede ser una persona de cincuenta años, que puede ser un joven, puede ser una mujer... No podría definirte ningún tipo de perfil del animador o de la animadora, porque es muy variopinto... (E04)).

Por otro lado, se asoció el sector a que pertenece la empresa que organiza la cena de Navidad con una mayor frecuencia de consumo de alcohol (...se consume yo creo que dos veces más si es una empresa, por ejemplo, de informática, o un consulting... esa gente, siempre bebe mucho... (E05)) y de otras sustancias (...es más normal en las empresas del sector servicios los viajes al baño... (E05)).

\section{Subagrupaciones}

En el presente estudio se detectó un fenómeno de agrupación entre pares, formando subgrupos dentro de aquellas mesas con un número elevado de asistentes (...a partir de quince o veinte ya se hacen grupitos. Sí. Es normal... (E06)), caracterizados por una mayor euforia y niveles más altos de consumo de alcohol que el resto de la mesa (...en ciertas zonas que se han juntado 5 ó 6, que van a, que está pidiendo mucho, y venga, que no falte de nada... (E02)).
En los subgrupos que muestran una mayor desinhibición predomina la presencia de gente más joven (...las conversaciones son divertidas, alegres, desinhibidas ... y existe mayor interrelación entre ellos... (0:R02)). Además de la edad, los profesionales se refieren, como razones de agrupación, a las propias relaciones entre los compañeros de trabajo, tanto por afinidad (...hay unos grupitos, que a lo mejor se juntan entre ellos y se ponen ya en una parte donde son más amenos o son más afines a ellos mismos... (E08)), como por evitación (...tenemos cenas, donde hay gente que no se tragan unos a otros, siempre se hacen pequeños grupos... (E01)), y dicha agrupación, según los entrevistados, no se asocia necesariamente con el consumo de sustancias (...no creo que los que más beben se junten, y los que más rayas se hacen se junten... (E03)), aunque las observaciones recogidas indican que sí que puede existir esa relación (...hay un subgrupo más animado que los demás, que son los que luego al salir afirman haber consumido "de todo"... (0:R11)).

\section{Alcohol y tráfico}

El $60,13 \%$ de los encuestados manifestó que el momento preferido para consumir tanto alcohol como sustancias ilegales es tras la cena (...suelen pedirse una copa, una copa o dos... (E02)), mientras que un $39,41 \%$ y un $16,6 \%$ percibian que era durante y antes de la cena respectivamente cuando los comensales llevaban a cabo dichos consumos. Tras la cena, un $81,6 \%$ de los encuestados había decidido continuar la fiesta, mientras que un $14,3 \%$ prefería ir a su casa.

Los hosteleros han destacado unánimemente en sus discursos una reducción del consumo de alcohol debida a los efectos que causa en la conducción (... si que se nota algo el consumo de alcohol por miedo a lo del carné por puntos, a conducir... (E04)). Incluso se observó que los mismos comensales tenían cierta precaución a la hora de seguir sirviendo alcohol a sus compañeros de mesa (...antes de servirle vino a un compañero uno le dice: "¿Has venido en coche? No. Pues entonces te sirvo"... (0:R02)).

En contraposición al caso del alcohol, los entrevistados inciden en la poca concienciación existente respecto al consumo de otras sustancias (...el consumidor de sustancias psicoactivas ilegales tiene una cierta tranquilidad... (E03)).

Asimismo, destacan otras consecuencias asociadas al consumo de sustancias en las cenas navideñas de empresa, como las relacionadas con los efectos de las mismas (...las consecuencias negativas que todos vivimos de las cenas de empresa son bien visibles... (E03)) o con la desinhibición que producen (...se podría hacer un buen estudio sobre las relaciones sexuales no deseadas, o prácticas de riesgo... (E03)).

\section{El papel de la hostelería en la prevención del consumo de sustancias: medidas preventivas}

La conducta de los camareros suele ser muy tolerante respecto al consumo de alcohol, y el discurso de los profesionales de la hostelería coincide con este planteamiento (... lo que cada uno se meta, esto no es responsabilidad del hos- 
telero... (E03)). Los responsables de los establecimientos hosteleros manifiestan tener muy claro su papel respecto al consumo de alcohol (... a nosotros no nos interesa. Yo tengo que abogar por mi empresa y por mi trabajo. Yo, a mí no me interesa que se haga ninguna campaña agresiva... (E06)). De hecho, incluso los mismos camareros en ocasiones llevan a cabo conductas de consumo de alcohol durante la cena (...los camareros definitivamente se han unido a la fiesta... beben vino tinto... (0:R06)). Además, señalan que los consumos realmente problemáticos aparecen tras la cena, y en lugares diferentes al propio restaurante (...lo peligroso viene a partir de la sobremesa... o sea, que el restaurante también cuenta, pero cuenta sobre todo, lo que se haga después, claro... (E04)).

Los profesionales de la hostelería proponen diversas alternativas como posibles intervenciones para prevenir el consumo abusivo de alcohol y otras drogas en las cenas navideñas de empresa y sus consecuencias asociadas, tales como:

a) Medios de transporte adecuados para estos eventos (...lo ideal sería que esa gente cogiera un taxi, o como hacen, que uno no beba... (E05)).

b) Una mayor especialización de las fuerzas de seguridad del Estado sobre detección de sustancias ilegales en controles de carretera y en la vía pública (...a mi lo que siempre me ha sorprendido de las fuerzas y cuerpos de seguridad, que deberían estar superespecializados... (E03)).

c) Restricción de horarios para el servicio de alcohol (...a lo mejor en el tema de horarios, el tema de prohibir la bebida a partir de cierta hora... (E02)).

d) Subida de precios del alcohol (...subir los precios, como el tabaco. Subir el precio un doscientos por cien. $Y$ entonces, pedirte una cerveza, y no pedirte dos... (E05)).

\section{DISCUSIÓN}

Los resultados del presente estudio muestran que las cenas navideñas de empresa constituyen un marco para el consumo recreativo de alcohol y otras drogas por parte de los asistentes a las mismas, con una serie de características similares al consumo recreativo de los jóvenes en zonas de ocio, al tratarse de grandes cantidades de consumo concentradas en un breve periodo de tiempo ${ }^{9,37}$. Además, a partir del discurso de los entrevistados y las observaciones realizadas, se han detectado una serie de características contextuales y personales que coinciden con algunos de los factores de riesgo para el consumo de drogas ${ }^{38}$ ya identificados por trabajos previos $^{39}$.

Se trata de un evento donde se reúnen numerosas personas, lo que facilita el consumo de alcohol, tal y como corroboran estudios previos que han demostrado que la agrupación favorece la frecuencia y cantidad de ingesta de bebidas alcohólicas ${ }^{40}$. Además, tal y como señaló Bruun ${ }^{41}$, dentro del grupo es permisible beber más que los demás, pero nunca beber menos.

Sin embargo, se trata de una situación que requiere de una aproximación preventiva adaptada, ya que la principal singularidad de estos eventos reside en el cambio de roles entre jóvenes y adultos, en contraposición al modelo de imitación de la conducta que los jóvenes llevan a cabo a partir de sus referentes adultos ${ }^{42,43}$. En este caso son los adultos quienes toman el ejemplo de los más jóvenes e inician un consumo de sustancias. Los adultos, además, instrumentalizan el consumo como una vía de rejuvenecimiento y de búsqueda de sensaciones, especialmente las de euforia y desinhibición; éstas, tal y como indican investigaciones previas ${ }^{44,45}$, constituyen unas de las motivaciones principales para iniciar un consumo de sustancias.

Uno de los resultados obtenidos en el estudio es un mayor consumo de sustancias en hombres que en mujeres, en consonancia con los datos de la última Encuesta Domiciliaria sobre Alcohol y Drogas del año $2005^{46}$, que indican que la extensión del uso de drogas en España durante dicho año fue bastante mayor en los hombres que en las mujeres, especialmente en el caso de las drogas de comercio ilegal. Sin embargo, los profesionales de la hostelería destacan que las mujeres también tienen altos índices de consumo aunque lo ocultan, tal vez debido a un proceso de estigmatización social ${ }^{47,48,49}$.

Otro de los resultados destacados de la presente investigación es el descenso del consumo de alcohol en las cenas navideñas de empresa respecto a años anteriores según los hosteleros. Este descenso se corresponde con el aumento de la concienciación de los ciudadanos con respecto a la mezcla de alcohol y conducción, que unido al refuerzo de los controles de alcoholemia en los periodos navideños ${ }^{50}$, puede haber contribuido al descenso de los accidentes de tráfico en el año $2007^{51}$. Los profesionales de la hostelería manifiestan que los asistentes de estas celebraciones navideñas no parecen percibir el consumo de sustancias ilegales como un peligro potencial sobre la conducción, si bien cualquier otra droga, fármaco o sustancia ingerida que afecte al sistema nervioso central puede reducir notablemente las facultades de los conductores ${ }^{52}$. El presente estudio, por tanto, ha puesto de manifiesto la importancia que tienen los profesionales del mundo hostelero como informadores clave.

Sin embargo, el papel de la hostelería en la prevención del consumo de alcohol y otras drogas es contradictorio, ya que en muchas ocasiones las intervenciones preventivas pueden jugar un papel contrario a sus intereses empresariales. Además, según el trabajo de Rodríguez-Martos ${ }^{53}$, la mayor parte de trabajadores de bares y restaurantes ignoran el contenido alcohólico de lo que dispensan, y no suelen conocer ni sentirse partícipes del daño que las consumiciones pueden producir en sus clientes.

El trabajo conjunto de los propietarios de bares, junto con el personal de los mismos pueden ayudar a crear entornos que potencien las ventajas económicas y sociales mini- 
mizando los riesgos que implican el consumo de drogas recreativas, el alcohol, la violencia, los accidentes y otras amenazas para la salud ${ }^{6}$. Por tanto, sin su colaboración, parece muy difícil que cualquier intervención preventiva pueda tener éxito (... las medidas preventivas dependen más del grado de responsabilidad social que tenga cada empresa que con sus medidas que están en el contexto de la autorregulación. No se pueden imponer medidas preventivas... (E03)).

\section{LIMITACIONES DEL ESTUDIO}

Los estudios exploratorios son necesarios para determinar las características de un fenómeno concreto, y proporcionar, de esta manera, un mejor enfoque en estudios posteriores, si bien cuentan con algunas limitaciones en cuanto a la generalización de los resultados obtenidos, fundamentalmente debidas al carácter preliminar y descriptivo de una aproximación exploratoria.

Durante el trabajo de campo surgieron dificultades respecto a las condiciones de realización del trabajo de campo. Los patrones de consumo sólo pueden ser registrados cuando se trata de conductas repetidas y regulares, y en el caso del presente estudio, las observaciones se llevaron a cabo en situaciones donde las interacciones son constantes y rápidas $y$, por tanto, dificultan el trabajo de los observadores. Esta potencial limitación se subsanó formando equipos de dos personas para realizar cada observación, recogiendo notas por separado y realizando posteriormente una puesta en común (cross checks).

Asimismo, las dificultades previstas durante la administración de las encuestas de estimación rápida, debido a las reticencias a participar en las mismas, y al estado de ebriedad de algunos de los encuestados, se subsanaron con la formación previa para los trabajadores de campo sobre administración de encuestas.

Los responsables de dos de los establecimientos donde se pretendia realizar la observación, tras ser informados del propósito de la investigación, también plantearon ciertas reticencias, tras lo que los trabajadores de campo abandonaron el local.

Pese a estas limitaciones, el estudio cuenta con los estándares de validez y fiabilidad que han sido establecidos para este tipo de estudios en la literatura ${ }^{54}$.

\section{AGRADECIMIENTOS}

Este estudio ha sido financiado por la Fundación de la Comunidad Valenciana para el Estudio, Prevención y Asistencia a las Drogodependencias de la Conselleria de Sanitat de la Generalitat Valenciana. Los autores quieren expresar su más sincero agradecimiento a las trabajadoras de campo Remedios Aguilar, Susana Chorén, Thais Dus, Iris Fernández,
Magdalena Jabazín, Rocío Molina, así como a Marina Ferrandis, Vanesa Navarro, Remedios García y los trabajadores del ámbito hostelero que han colaborado en esta investigación.

\section{REFERENCIAS}

1. Becoña E. Los adolescentes y el consumo de drogas. Papeles del Psicólogo 2000; 77: 25-32.

2. Riley SC, Blackman, G. Between prohibitions: Patterns and meanings of magic mushroom use in the UK. Subst Use Misuse 2008; 43: 55-71.

3. Wanjek B, Rosendahl J, Strauss B, Gabriel HH. Doping, drugs and drug abuse among adolescents in the State of Thuringia (Germany): Prevalence, knowledge and attitudes. Int J Sports Med 2007; 28: 346-53.

4. Hopper JW, Su ZH, Looby AR, Ryan ET, Penetar DM, Palmer CM, et al. Incidence and patterns of polydrug use and craving for ecstasy in regular ecstasy users: An ecological momentary assessment study. Drug Alcohol Depend 2006; 85: 221-35.

5. Goldstein LH, Mordish Y, Abu-Kishak I, Toledano M, Berkovitch M. Acute paralysis following recreational MDMA (Ecstasy) use. Clin Toxicol 2006; 44: 339-41.

6. Bellis MA, Hughes K. Consumo recreativo de drogas y reducción de daños en la vida nocturna global. Adicciones 2003; 15: 289-305.

7. Laespada MT, Salazar L. Las actividades no formalizadas de los jóvenes. En Elzo J, Orizo FA, González-Anleo J, González P, Laespada MT, Salazar L editores. Jóvenes españoles '99. Madrid: Fundación Santa María; 1999. p. 355-400.

8. Rodríguez J, Agulló E, Agulló MS. Jóvenes, fin de semana y uso recreativo de drogas: evolución y tendencias del ocio juvenil. Adicciones 2003; 15 supl. 2: 7-34.

9. Drogomedia.com. Drogas de sintesis en Euskadi. (Fecha de acceso: 4-11-2007). Disponible en: http://www.drogomedia.com/ dossiers1_lasclaves.php?dossier $=9$

10. 20 Minutos. Lanzan una campaña para evitar las borracheras en las cenas de empresa. (Fecha de acceso: 10 de marzo de 2008). Disponible en: http://www.20minutos.es/noticia/321405/0/ alcohol/cenas/empresa/

11. Glindemann KE, Wiegand DM, Geller ES. Celebratory drinking and intoxication - A contextual influence on alcohol consumption. Environ Behav 2007; 39: 352-66.

12. Klein G, Gardiwal A, Schaefer A, Panning B, Breitmeier D. Effect of ethanol on cardiac single sodium channel gating. Forensic Sci Int 2007; 171: 131-35.

13. Aasebo W, Erikssen J, Jonsbu J, Stavem K. ECG changes in patients with acute ethanol intoxication. Scand Cardiovasc J 2007; 41: 79-84.

14. Uyarel H, Ozdol C, Gencer AM, Okmen E, Cam N. Acute alcohol intake and OT dispersion in healthy subjects. J Stud Alcohol 2005; 66: 555-8.

15. Frost $L$, Vestergaard P. Alcohol and risk of atrial fibrillation or flutter - A cohort study. Arch Intern Med 2004; 164: 1993-8.

16. Steinbigler $P$, Haberl $R$, Konig $B$, Steinbeck G. P-wave signal averaging identifies patients prone to alcohol-induced paroxysmal atrial fibrillation. Am J Cardiol 2003; 91: 491-4.

17. Raty S, Sand J, Alho H, Nordback I. Alcoholic, but not biliary, pancreatitis varies seasonally in occurrence. Scand J Gastroenterol 2003; 38: 794-7. 
18. Makela P, Martikainen P, Nihtila E. Temporal variation in deaths related to alcohol intoxication and drinking. Int J Epidemiol 2005; 34: 765-71.

19. Poikolainen $K$, Leppanen $K$, Vuori E. Alcohol sales and fatal alcohol poisonings: a time-series analysis. Addiction 2002; 97:1037-40.

20. Farmer $\mathrm{CM}$, Williams AF. Temporal factors in motor vehicle crash deaths. Inj Prev 2005; 11: 18-23.

21. Kalediene $R$, Petrauskiene J. Inequalities in daily variations of deaths from suicide in Lithuania: Identification of possible risk factors. Suicide Life Threat Behav 2004; 34:138-46.

22. Bradvik L, Berglund M. A suicide peak after weekends and holidays in patients with alcohol dependence. Suicide Life Threat Behav 2003; 33: 186-91.

23. Charbonney E, Sztajzel JM, Poletti PA, Rutschmann O. Paroxysmal atrial fibrillation after recreational marijuana smoking: another "holiday heart"? Swiss Med Wkly 2005; 135: 412-4.

24. Trotter RT, Needle RH, Goosby E, Bates C, Singer M. A Methodological Model for Rapid Assessment, Response and Evaluation: the RARE Program in Public Health. Field methods 2001;13:137-59.

25. Stimson GV, Fitch $\mathrm{C}$, Rhodes T. The rapid assessment and response guide on injecting drug use. Geneva: World Health Organization Substance Abuse Department; 1998.

26. Rhodes T, Ball A, Stimson GV, Kobyshcha Y, Fitch C, Pokrovsky, V, et al. HIV infection associated with drug injecting in the newly independent states, eastern Europe: the social and economic context of epidemics. Addiction 1999; 94: 1323-36.

27. Patton MQ. Qualitative evaluation and research methods (2nd ed.). Newbury Park, CA: Sage Publications; 1990.

28. Grov C, Parsons JT, Bimbi DS. Sexual Risk Behavior and Venues for Meeting Sex Partners: An Intercept Survey of Gay and Bisexual Men in LA and NYC. AIDS Behav 2007; 11: 915-26.

29. Rotheram-Borus MJ, Mann T, Newman PA, Grusky O, Frerichs RR, Wight RG, Kuklinski, M. A street intercept survey to assess HIVtesting attitudes and behaviors. AIDS Educ Prev 2001; 3: 229 - 38.

30. Miller KW, Wilder LB, Stillman FA, Becker DM. The feasibility of a street-intercept survey method in an African-American community. Am J Public Health 1997; 87: 655-8.

31. Lewis-Beck MS, Bryman A, Liao TF. The Sage Encyclopedia of Social Science Research Methods. Vol. 2. London: Sage Publications, 2004.

32. Needle $R$, Trotter $R$, Singer $M$, Bates $C$, Page JB, Metzger $D$ et al. Rapid Assessment of the HIVIAIDS Crisis in Racial and Ethnic Minority Communities: An Approach for Timely Community Interventions. Am J Public Health 2003; 93: 970-9.

33. Glaser BG, Strauss AL. The Discovery of Grounded Theory: Strategies for Qualitative Research. Chicago: Aldine Publishing Company; 1967.

34. Taylor S, Bogdan R. Introducción a los métodos cualitativos de investigación. Barcelona: Paidós; 1987.

35. Single $E_{1}$ Pomeroy $H$. Drinking and Setting: a season for all things. En Peele S, Grant M, editores. Alcohol and Pleasure. Philadelphia: Brunner/Mazel, Taylor and Francis; 1999.

36. Navarro J. El consumo de drogas en la Comunidad Valenciana 2006. Madrid: Conselleria de Sanidad, Generalitat Valenciana y Fundación de Ayuda contra la Drogadicción; 2007.

37. Calafat A. El abuso de alcohol de los jóvenes en España (Editorial). Adicciones 2007; 19: 217-224.
38. Muñoz-Rivas MJ, Graña $J L$, Cruzado JA. Factores de riesgo en drogodependencias: Consumo de drogas en adolescentes. Madrid: Sociedad Española de Psicología Clínica, Legal y Forense; 2000.

39. Clayton RR. Transitions in drug use: Risk and protective factors. En Glantz M, Pickens R. Vulnerability to drug abuse (pp.). Washington, DC: American Psychological Association; 1992. p. 15-51.

40. Wilsnack SC, Murray MM. Alcohol abuse and stigma Comunicación presentada en "Stigma and Global Health: an International Conference». Bethesda, 5-7 septiembre de 2001.

41. Bruun K. Drinking behaviour in small groups. Helsinki: Finnish Foundation for Alcohol Studies; 1959.

42. Bandura A. Social foundations of thought and action: A social cognitive theory. New Jersey: Prentice Hall, 1986.

43. Whiteman SD, Christiansen A. Processes of Sibling Influence in Adolescence: Individual and Family Correlates. Fam Relat 2008; 57: 24-34.

44. Magid V, MacLean MG, Colder CR. Differentiating between sensation seeking and impulsivity through their mediated relations with alcohol use and problems. Addict Behav 2007; 32: 2046-61.

45. Leeman RF, Toll BA, Volpicelli JR. The Drinking-Induced Disinhibition Scale (DIDS): A measure of three types of disinhibiting effects. Addict Behav 2007; 32: 1200-19.

46. Observatorio Español sobre Drogas. Encuesta Domiciliaria sobre Alcohol y Drogas en España 2005. Madrid: Delegación del Gobierno para el Plan Nacional sobre Drogas; 2005.

47. Valderrama JC, Tortajada S, Alapont L, Vidal A, Perez MJ, Castellano $M$ et al. Rapid Assessment of Drug Abuse Prevention Needs for Youth in Small Spanish Municipalities: Coping with Resource Limitations. J Drug Issues 2006; 36: 29-52.

48. Gómez J. El alcoholismo femenino, una verdad oculta. Trastornos Adictivos 2006; 8: 251-60.

49. Megias E, Rodríguez E, Megías I, Navarro J. La percepción social de los problemas de drogas en España 2004, Madrid: Fundación de Ayuda contra la Drogadicción; 2005.

50. Murcia refuerza sus acciones de prevención en Navidad. Diario Médico. Jueves 14 de diciembre de 2006.

51. Dirección General de Tráfico. Accident rate assessment: Year 2007, provisional data. (Fecha de acceso: 4 de marzo de 2008). Disponible en URL: http://www.dgt.es/was6/portal/contenidos/ documentos/seguridad_vial/estudios_informes/est_info_ segVial055.pdf

52. Hunter CE, Lokan RJ, Longo MC, White JM, White MA. The prevalence and role of alcohol, cannabinoids, benzodiazepines and stimulants in non-fatal crashes. Adelaide, South Australia: Forensic Science, Department for Administrative and Information Services, South Australia, 1998.

53. Rodríguez-Martos A. Prevención de lesiones atribuibles al alcohol en el marco de una política de reducción de daños. Trastornos Adictivos 2002; 4: 95 - 108.

54. Trotter RT, Needle RH. RARE Methods resource guide. Washington DC: Department of Health and Human Services; 2000. 